\title{
Direct measurement of the beam deflection angle using the axial $B$-dot field
}

\author{
Xiaozhong He, Kaizhi Zhang, and Qin Li \\ Institute of Fluid Physics, Chinese Academy of Engineering of Physics, Mianyang, Sichuan, China
}

(Received 2 October 2010; published 31 May 2011)

\begin{abstract}
Beam position monitors are an important diagnostics tool for particle accelerator operation and related beam dynamics research. The measurement of the beam deflection angle, or moving direction of a charged particle beam with respect to the beam pipe axis, can provide useful additional information. Beam monitors sensitive to the beam's azimuthal $B$-dot field (sometimes referred as $B$ dots) are used to measure the displacement (position) of the beam centroid, as the beam generates a dipole term of the azimuthal magnetic field. Similarly, a dipole term of the axial magnetic field will be generated by the beam moving in a direction not parallel to the axis of the beam pipe. In this paper, a new method using the axial $B$-dot field is presented to measure the beam deflection angle directly, including the theoretical background. Simulations using the MAFIA numerical code have been performed, demonstrating a good agreement to the new established analytical model.
\end{abstract}

DOI: 10.1103/PhysRevSTAB.14.052804

PACS numbers: 06.30.Gv

\section{INTRODUCTION}

In accelerators a charged particle beam propagates inside an evacuated, conducting beam pipe, and undergoes a transverse motion due to the magnetic guiding fields. An accurate measurement of the transverse beam displacement is very important for understanding the underlying beam physics of the transverse particle motion, i.e., the so-called beam optics. For example, in linear induction accelerators, the measurement of the beam transverse displacement can be used to determine how to correct the beam center position error to suppress the transverse emittance growth due to cockscrew effect [1].

Additionally, when a beam of high intensity propagates in the conducting beam pipe, it interacts with the surroundings and the likely existing background plasma may encounter instabilities such as beam breakup effects [2] and ion hose instabilities [3,4]. Usually, the transverse beam displacement is measured at a series of locations along the beam pipe, which gives the beam orbit information of the beam centroid, e.g., to understand beam instabilities based on the amount of growth rate. The beam centroid position measurement can be performed by using azimuthal $B$-dot monitors [5-9].

The principle of an azimuthal $B$-dot beam positing monitor is shown in Fig. 1.

The time integrated signal of the wire-loop electrode is proportional to the azimuthal magnetic field. For a displaced line current inside a long conducting cylindrical pipe, its azimuthal magnetic field at the inner wall

Published by the American Physical Society under the terms of the Creative Commons Attribution 3.0 License. Further distribution of this work must maintain attribution to the author(s) and the published article's title, journal citation, and DOI. (more specifically speaking, infinitely close to the inner wall) of the conducting pipe can be expressed [10-12]:

$$
B_{\theta}(r=b, \theta)=\frac{\mu_{0} I}{2 \pi b} \frac{1-\rho^{2}}{1+\rho^{2}-2 \rho \cos (\theta-\varphi)},
$$

where $\boldsymbol{I}$ is the beam current, $b$ is the inner radius of the beam pipe, $\rho=a / b$ is the normalized magnitude of the beam displacement, and $(a, \varphi)$ is the transverse beam position in cylindrical coordinates system $(r, \theta)$.

At the four locations $x+, y+, x-$, and $y-$ of the loop electrodes (see Fig. 1), Eq. (1) has a very simple form in approximation for small displacement $(\rho \ll 1)[12]$ :

$$
\begin{gathered}
B_{\theta}(r=b, \theta=0)=B_{0}(1+2 \rho \cos \varphi) \\
B_{\theta}(r=b, \theta=\pi / 2)=B_{0}(1+2 \rho \sin \varphi) \\
B_{\theta}(r=b, \theta=\pi)=B_{0}(1-2 \rho \cos \varphi) \\
B_{\theta}(r=b, \theta=3 \pi / 2)=B_{0}(1-2 \rho \sin \varphi),
\end{gathered}
$$

where $B_{0}=\mu_{0} I / 2 \pi b$ is the magnetic field at the inner wall of beam pipe for a centered beam. One can conclude that the magnetic field difference between the $x+$ direction and the $x$ - direction is proportional to the displacement in the $x$ direction, and vice versa for the vertical coordinate.

Therefore the azimuthal $B$-dot beam position monitor of four small loop electrodes at the $x+, y+, x-$, and $y-$ locations can measure any transverse beam displacement in the $x-y$ plane.

As the beam centroid offset is varying along the $z$ axis, the related azimuthal magnetic field also varies. The relation between azimuthal and axial magnetic field components is given by Maxwell's equation:

$$
\frac{\partial B_{z}}{r \partial \theta}-\frac{\partial B_{\theta}}{\partial z}=\frac{\partial E_{r}}{c^{2} \partial t} .
$$




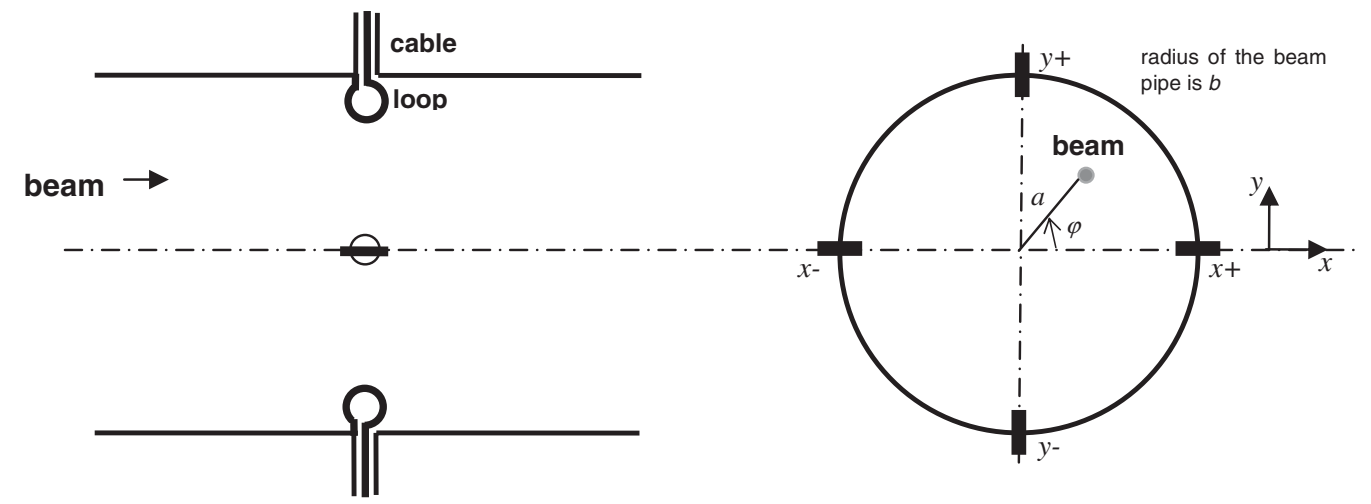

FIG. 1. Diagram of azimuthal $B$ dot.

Equation (6) is only valid for fields near the inner surface of the beam pipe and it assumes that the radial beam current density $j_{r}$ in this region is very small and its contribution to the magnetic fields can be neglected.

According to Eqs. (2)-(5), the derivative of the azimuthal magnetic field with respect to the axial position $z$ is proportional to the beam deflection angle, $x^{\prime}=$ $d(a \cos \varphi) / d z, y^{\prime}=d(a \sin \varphi) / d z$. This hints that there is an analytic expression which relates the axial magnetic field to the beam deflection angle, and there is a method to measure the beam deflection angle directly. A simple, direct measurement of the beam deflection angle will provide additional, useful information about the beam dynamics in accelerators.

In Sec. II we will establish the theory, explaining the link between axial magnetic fields and beam deflection angle. Section III discusses various configurations of the axial $B$-dot wire loops to measure the beam deflection angle. In Sec. IV we compare our analytical investigations with numerical simulations using the MAFIA code, often used for solving Maxwell's equation problems.

\section{THEORY}

\section{A. Approximation of a slow temporal variation and a small beam displacement}

Consider a slow temporal variation of the beam, i.e., the contribution of the displacement current $d E / d t$ to $B_{\theta}$ is small, and furthermore only small displacements $(\rho \ll 1)$ of the beam inside a long conducting pipe. Shokair derived a closed expression for the azimuthal magnetic field, taking a $z$ dependence of the nonlocal beam current into account [10]:

$$
B_{z}(r=b, \theta, z)=\frac{\mu_{0} I}{2 \pi}\left(\frac{1}{b}+\frac{2}{b^{2}} \int_{-\infty}^{\infty} d k \frac{e^{-i k z}}{2 \pi} \Gamma(k b) \tilde{u}(k, \theta)\right),
$$

where $\Gamma$ is defined as Eqs. (8)-(10)

$$
\Gamma(x)=\frac{x K_{1}(x)}{x Q_{1}(x)+P_{1}(x)},
$$

$$
Q_{n}(x)=\frac{-x}{\pi} \int_{0}^{2 \pi} d \psi \sin (\psi) \sin (n \psi) K_{0}[x \sqrt{2-2 \cos (\psi)}]
$$

$$
\begin{aligned}
P_{n}(x)= & 1+\frac{x}{2 \pi} \int_{0}^{2 \pi} d \psi \cos (\psi) \\
& \times \sqrt{2-2 \cos (\psi)} K_{1}[x \sqrt{2-2 \cos (\psi)}],
\end{aligned}
$$

and $\tilde{u}(k, \theta)$ is the Fourier transform of $u(z, \theta)=\rho(z) \times$ $\cos [\varphi(z)-\theta], K(x) n$ is the modified Bessel function of second kind.

Assuming a slow temporal variation of the displacement current, the right-hand side of Eq. (6) approximates to zero. Combining Eqs. (6) and (7) gives

$$
F T\left[\frac{\partial B_{z}}{r \partial \theta}\right]=-i k \tilde{u}(k, \theta) \frac{2 \mu_{0} I}{b^{2}} \Gamma(k b) .
$$

Applying the inverse Fourier transform to Eq. (11) yields

$$
\frac{\partial B_{z}(r=b, \theta, z)}{\partial \theta}=\frac{\mu_{0} I}{2 \pi b} \int_{-\infty}^{\infty} \Gamma(k b) \frac{\partial \tilde{u}}{\partial z}(k, \theta) e^{-i k z} d k,
$$

where $\frac{\partial \tilde{u}}{\partial z}(k, \theta)$ is the Fourier transform of $\frac{\partial u}{\partial z}(z, \theta)$ with respect to $z$.

From (12) we derive an expression for $B_{z}$,

$$
B_{z}(r=b, \theta, z)=\frac{-\mu_{0} I}{\pi b} \int_{-\infty}^{\infty} \Gamma(k b) \frac{\partial \tilde{v}}{\partial z}(k, \theta) e^{-i k z} d k,
$$

where $\frac{\partial \tilde{v}}{\partial z}(k, \theta)$ is the Fourier transform of $\frac{\partial v}{\partial z}(z, \theta)$ with respect to $z$, and $v(z, \theta)=\rho(z) \sin [\varphi(z)-\theta]$.

If we symbolize the inverse Fourier transform of $\Gamma(k b)$ as $g(z)$, Eq. (13) can be represented in the form of a convolution integral:

$$
B_{z}(r=b, \theta, z)=\frac{-\mu_{0} I}{\pi b} \int_{-\infty}^{\infty} \frac{\partial v}{\partial z^{\prime}}\left(z^{\prime}, \theta\right) g\left(z-z^{\prime}, b\right) d z^{\prime} .
$$

For the case of the $x+, x-, y+$, and $y-$ direction, we get the results

$$
B_{z}(r=b, \theta=0, z)=\frac{-\mu_{0} I}{\pi b} \int_{-\infty}^{\infty} y^{\prime}\left(z^{\prime}\right) g\left(z-z^{\prime}, b\right) d z^{\prime}
$$




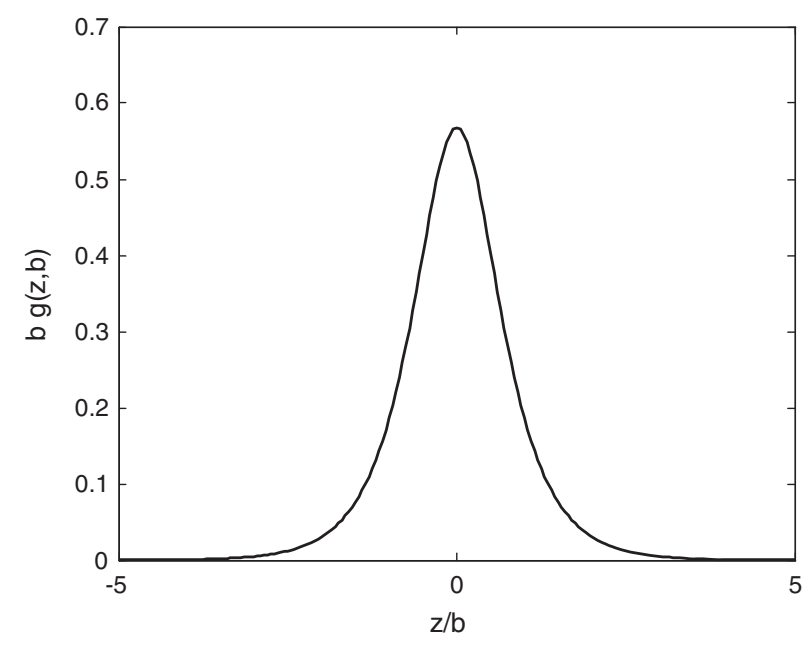

FIG. 2. The value of function $b g(z, b)$.

$$
\begin{gathered}
B_{z}(r=b, \theta=\pi, z)=\frac{\mu_{0} I}{\pi b} \int_{-\infty}^{\infty} y^{\prime}\left(z^{\prime}\right) g\left(z-z^{\prime}, b\right) d z^{\prime} \\
B_{z}(r=b, \theta=\pi / 2, z)=\frac{\mu_{0} I}{\pi b} \int_{-\infty}^{\infty} x^{\prime}\left(z^{\prime}\right) g\left(z-z^{\prime}, b\right) d z^{\prime} \\
B_{z}(r=b, \theta=3 \pi / 2, z)=\frac{-\mu_{0} I}{\pi b} \int_{-\infty}^{\infty} x^{\prime}\left(z^{\prime}\right) g\left(z-z^{\prime}, b\right) d z^{\prime},
\end{gathered}
$$

where $x^{\prime}$ and $y^{\prime}$ are the beam deflection angles in the $x$ and the $y$ direction, respectively. Equations (15)-(18) show that $B_{z}$ is proportional to the weighted average of the beam deflection angle, with $g(z, b)$ as a weighting function.

The function $g(z, b)$ times $b$ gives the longitudinal extent of the axial magnetic field due to the beam deflection. The value of $g(z, b)$ times $b$ can be evaluated numerically [10], see the plot of $g(z, b)$ times $b$ in Fig. 2 over a longitudinal range $z$ of several beam pipe radii $b$.

\section{B. Approximation of a small ratio of azimuthal to axial image current}

In the case of pulsed beam propagating with small displacement $\rho \ll 1$ and small deflection angle $x^{\prime} \ll 1$ $y^{\prime} \ll 1$, the ratio of azimuthal image current $\left(J_{\theta}\right)$ and axial image current $\left(J_{z}\right)$ is small, i.e., the image charges on the inner surface of the conducting beam pipe travel with the same velocity as the charged beam

$$
\sigma \beta c=J_{z}
$$

where $\sigma$ is density of the image charge density and $\beta c$ is the velocity of the bunched beam. Equation (19) relates to

$$
E_{r}(r=b, \theta)=B_{\theta}(r=b, \theta) c / \beta .
$$

Inserting Eq. (20) into Eq. (6) results in

$$
\frac{\partial B_{z}}{r \partial \theta}=\frac{\partial E_{r}}{c^{2} \partial t}+\frac{\partial B_{\theta}}{\partial z}=\frac{d B_{\theta}\left(r, \theta, z, t=t_{0}+z / \beta c\right)}{d z} .
$$

A point charge with charge $q$, traveling at relativistic velocity along a conducting beam pipe with circular cross section, generates an azimuthal magnetic field of [13]

$$
\begin{aligned}
B_{\theta}(r=b, \theta)= & \sum_{m=0}^{\infty} \frac{2 q a^{m}}{1+\delta_{m 0}} \cdot \frac{1}{4 \pi \varepsilon_{0} c} \delta(z-c t) \\
& \times \cos [m(\theta-\varphi)] \cdot \frac{2}{b^{m+1}},
\end{aligned}
$$

where $\delta_{m 0}=1$ if $m=0$ and $\delta_{m 0}=0$ if $m \neq 0$.

For a line charge with density of $\lambda(z-\beta c t)$, we need to replace $q \delta(z-c t)$ in Eq. (22) by $\lambda(z-\beta c t)$. Actually, Eq. (22) is equivalent to Eq. (1). Equation (22) holds only if $\rho$ and $\varphi$ are constant. For an arbitrary transverse displacement along $z$ and small beam deflection angle, the transverse displacement vary slowly with $z$, and $B$ is determined by the local transverse displacement (or in other words, the contribution of the $z$ dependence of nonlocal beam displacement is not significant); therefore Eq. (22) still holds. Inserting Eq. (22) into Eq. (21) yields the axial magnetic field

$$
\begin{aligned}
B_{z}(r=b, \theta)= & \sum_{m=1}^{\infty} \frac{\mu_{0} I(z-\beta c t)}{\pi b} \cdot \frac{a^{m-1}}{b^{m-1}}\left\{a^{\prime} \sin [m(\theta-\varphi)]\right. \\
& \left.-a \varphi^{\prime} \cos [m(\theta-\varphi)]\right\} .
\end{aligned}
$$

Neglecting higher order terms, Eq. (23) simplifies to

$$
\begin{gathered}
B_{z}(r=b, \theta=0)=2 B_{0}\left(-y^{\prime}-\frac{x^{\prime} y+y^{\prime} x}{b}\right) \\
B_{z}(r=b, \theta=\pi / 2)=2 B_{0}\left(x^{\prime}+\frac{x^{\prime} y+y^{\prime} x}{b}\right) \\
B_{z}(r=b, \theta=\pi)=2 B_{0}\left(y^{\prime}-\frac{x^{\prime} y+y^{\prime} x}{b}\right) \\
B_{z}(r=b, \theta=3 \pi / 2)=2 B_{0}\left(-x^{\prime}+\frac{x^{\prime} y+y^{\prime} x}{b}\right)
\end{gathered}
$$

The axial magnetic field difference between the $x+$ and the $x$ - direction is

$$
B_{z}(r=b, \theta=0)-B_{z}(r=b, \theta=\pi)=-4 B_{0} y^{\prime},
$$

and the axial magnetic field difference between the $y+$ and the $y$ - direction is

$$
B_{z}(r=b, \theta=\pi / 2)-B_{z}(r=b, \theta=3 \pi / 2)=4 B_{0} x^{\prime},
$$

where $B_{0}=\frac{\mu_{0} I(s-\beta c t)}{2 \pi b}$ is the magnetic field at the inner wall surface of the beam pipe for a centered beam.

Equations (24)-(27) provide a solution of the axial magnetic field dependence to the deflection angle for a 
pulse beam propagating with small beam displacement $(\rho \ll 1)$ and small deflection angles $\left(x^{\prime} \ll 1, y^{\prime} \ll 1\right)$, i.e. $B_{z} / B_{\theta} \ll 1$.

For relativistic beams both sets of equations, Eq. (15)(18) and Eq. (24)-(27), can be used to compute the axial magnetic field. The first set of equations, derived under the approximation of a slow temporal variation and small displacement of the beam, takes into account the contribution of a nonlocal beam current.

The latter set of equations neglects the nonlocal effects, but has the time dependence and higher accuracy because the multiplication term of beam deflection angle and normalized beam displacement is included. In the case where $x^{\prime}$ and $y^{\prime}$ vary slowly along the axial position (wavelength is larger than several beam pipe radii), the nonlocal effects are not significant, so the latter set of equations is applicable. In the case where the nonlocal effects are significant, the function $g(z, b)$ should be convoluted to Eqs. (24)-(27) to get more accurate results.

For nonrelativistic beams under the slow temporal variation approximation, i.e., for long bunches, Eqs. (15)-(18) are still valid. In the case of a nonrelativistic beam with short bunches, Eq. (21) still holds, but the time dependence in Eq. (22) becomes invalid. We need to modify the common term $B_{0}=\frac{\mu_{0} I(s-\beta c t)}{2 \pi b}$ in Eqs. (24)-(27) to account for the longitudinal bunch profile.

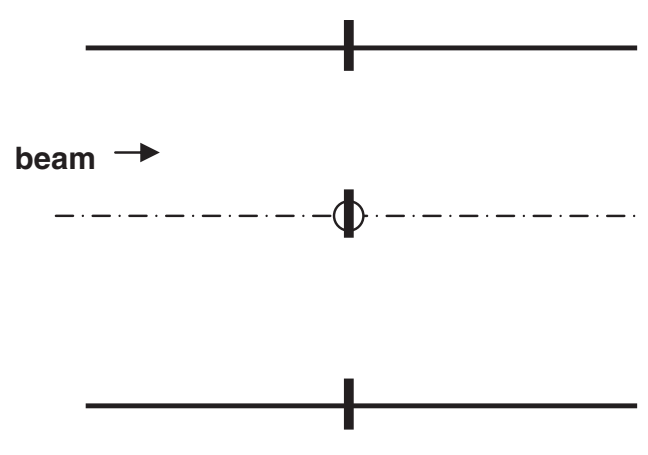

\section{AXIAL $B$-DOT CONFIGURATIONS FOR DIRECT MEASUREMENT OF THE BEAM DEFLECTION ANGLE}

In the case of relativistic or nonrelativistic beams having long bunches and with known longitudinal beam current profile $I(z-\beta c t)$, a direct measurement of the axial magnetic field can be realized simply by locating four wire-loop antennas at the $x+, x-, y+$, and $y$ - positions (see Fig. 3); similar to the azimuthal $B$-dot beam position monitor arrangement of Fig. 1, but here the loops are oriented to detect the axial $B$ field. Applying Eqs. (28) and (29) we simply can measure the beam deflection angles $x^{\prime}$ and $y^{\prime}$.

In the case the longitudinal bunch profile is unknown, we may combine axial and azimuthal $B$-dot wire loops in a symmetric arrangement at the same $z$ location, as shown in Fig. 4. The signals from the four wire loops detecting the azimuthal magnetic field may be used to measure the transverse beam displacement, as well as the longitudinal bunch profile, while the four wire loops detecting the axial magnetic field are used for the beam angle measurement.

In case of a nonrelativistic beam with short bunches we can use the same "double" configuration (Fig. 4), the common mode signal $B_{0}$ is measured by the azimuthal $B$-dot loop electrodes, required to determine the beam angle based on Eqs. (28) and (29) using the axial $B$-dot loop electrodes.

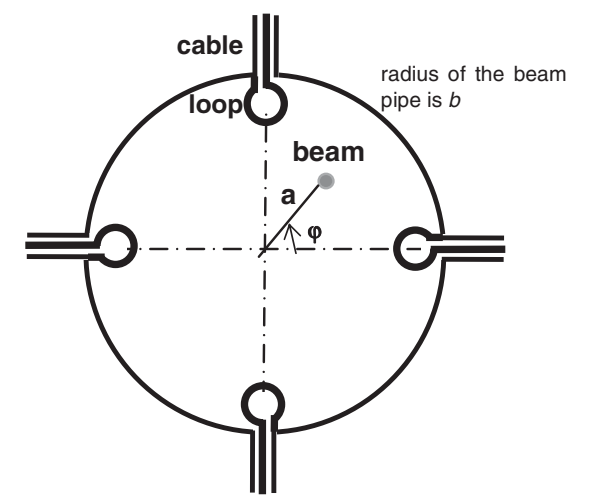

FIG. 3. Four wire loops to measure beam deflection angle.
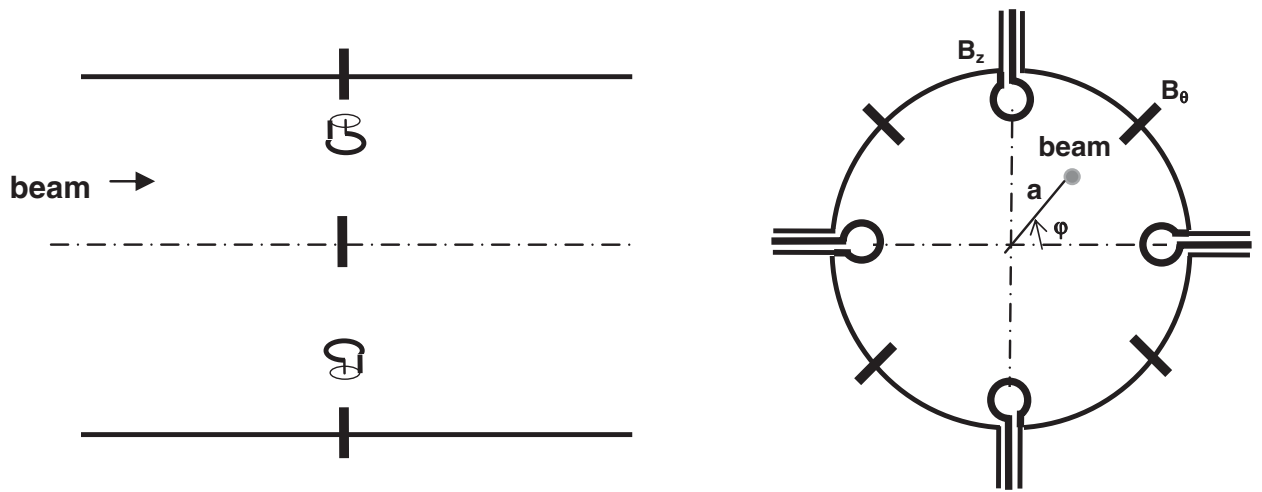

FIG. 4. Azimuthal $B$ dots and axial $B$ dots are composed to measure beam current, displacement, and beam deflection angle. 
Strictly speaking, nonlocal effects are always present, only in the case that the beam deflection angle varies slowly along $z$ (betatron wavelength is larger than several beam pipe radii), the beam deflection angle can be measured locally by using the axial $B$-dot method. If the betatron wavelength is short, the axial magnetic field has to be measured at several locations along $z$, and the beam angles can be determined by inverse convolution. This approach is similar to the method given in Shokair's paper [10], which measures the azimuthal magnetic field at a series of axial location and computes the beam displacement profile through inverse convolution.

\section{SIMULATIONS TO VERIFY THE THEORETICAL RESULTS}

A series of numerical simulations have been performed to verify the analytic approach.

\section{A. A relativistic beam inside a beam pipe of circular cross section along a lattice with four steering magnets}

\section{Geometry}

The beam pipe has a circular cross section of $147 \mathrm{~mm}$ diameter and is $10.5 \mathrm{~m}$ long. The four steering magnets are located at $3,4.5,6$, and $7.5 \mathrm{~m}$ distance from the beam entrance; these four locations are labeled C, D, E, and F in
Fig. 5, respectively. The magnetic fields are recorded in the $x-y$ plane at four locations $3.75,5.25,6.75$, and $9 \mathrm{~m}$, labeled $\mathrm{G}, \mathrm{H}, \mathrm{I}$, and $\mathrm{J}$.

\section{Parameters of injected beam}

The injected beam has a Gaussian temporal profile with standard deviation of $5 \mathrm{~ns}$. Its total charge is $20 \mu \mathrm{C}$ and its energy is $\mathrm{pc}=1 \mathrm{TeV}$. The transverse distribution is uniform and has a radius of $5 \mathrm{~mm}$. The beam is injected at the center, along the axis.

\section{Simulation software}

The MAFIA $S$ module is used to compute the magnetic field of steering coils. The TS3 module is used to self-consistently compute the fields in the presence of the beam.

\section{Boundary conditions}

The boundary conditions in the longitudinal direction (A and B plane in Fig. 5) are set to be waveguide. All modes with frequency less than $4 \mathrm{GHz}$ are included, and can propagate through the waveguide boundary.

\section{Simulation results}

The trajectory of the beam with respect to the beam pipe center is shown in Fig. 6. A closed, asymmetric beam bump

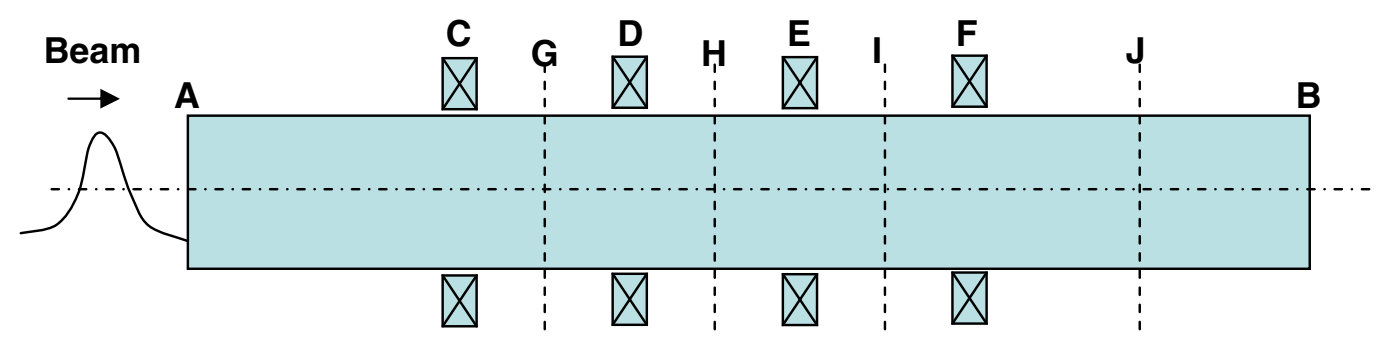

FIG. 5. Geometry of simulation in Sec. IVA.
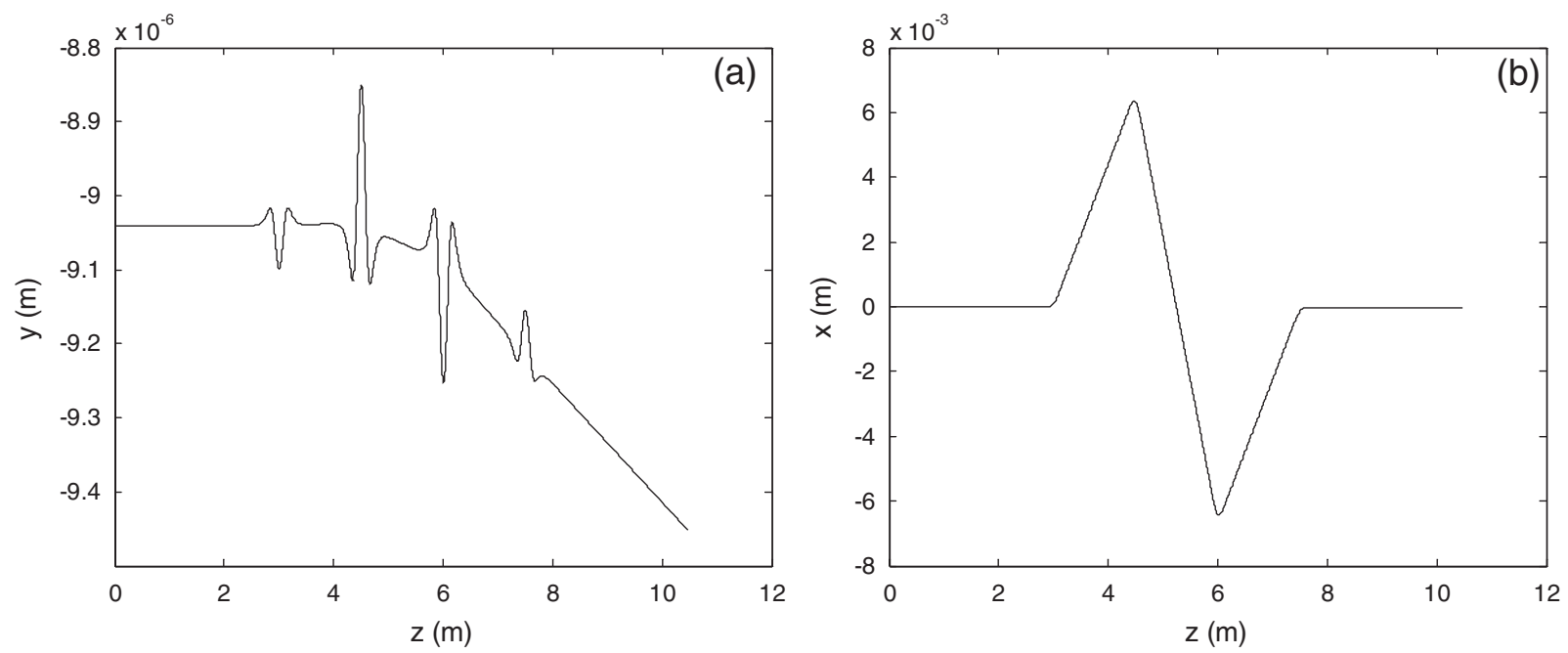

FIG. 6. (a) $y$ of the beam vs $z$, (b) $x$ of the beam vs $z$. 

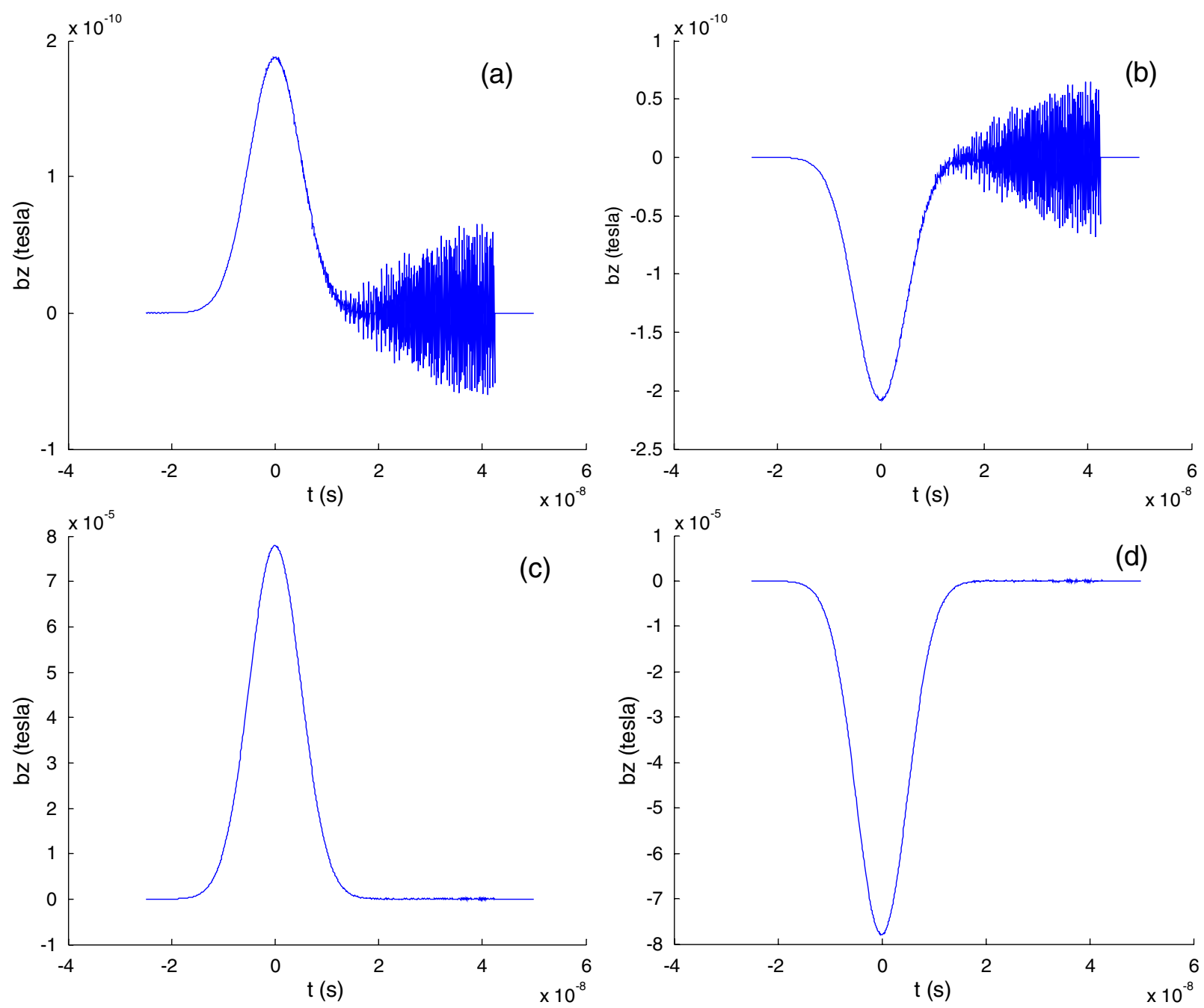

FIG. 7. (a) $B_{z}$ at $x=b, y=0$; (b) $B_{z}$ at $x=-b, y=0$; (c) $B_{z}$ at $x=0, y=b$, (d) $B_{z}$ at $x=0, y=-b$, where $b$ is the radius of the beam pipe. For all four plots $z=5.25 \mathrm{~m}$.

is performed in the horizontal plane; no deflection is applied in the vertical plane.

The axial magnetic fields at the " $\mathrm{H}$ " plane location are shown in Fig. 7, plotted for the four locations of the wire loops. The temporal profile of the axial magnetic field is Gaussian, and has the same standard variation of $5 \mathrm{~ns}$. Because it is not possible for MAFIA (or the authors cannot find a proper way) to include all modes in the waveguide boundary, the modes with intrinsic frequency higher than $4 \mathrm{GHz}$ are not included in the waveguide boundary. For these modes the simulation geometry acts like a cavity, so noise will stimulate these modes and lead to the oscillations of the magnetic field after the beam leaves the observation positions. Applying Eq. (29), we compute the beam deflection angle of $-8.78 \mathrm{mrad}$ at $z=5.25 \mathrm{~m}$ in the horizontal, and almost zero in the vertical plane. This is in very good agreement to the numerical result, showing a deflection angle of $-8.86 \mathrm{mrad}$ in the horizontal plane.

\section{B. A quasirelativistic beam inside a beam pipe of circular cross section along a lattice with four steering magnets and a solenoid}

\section{Geometry}

The physical layout is the same as in Sec. IVA, except a solenoidal magnet is added. The physical layout is shown in Fig. 8. The solenoid covers the longitudinal region from 0.6 to $9.6 \mathrm{~m}$. Beside recording the magnetic fields at the $\mathrm{G}$,

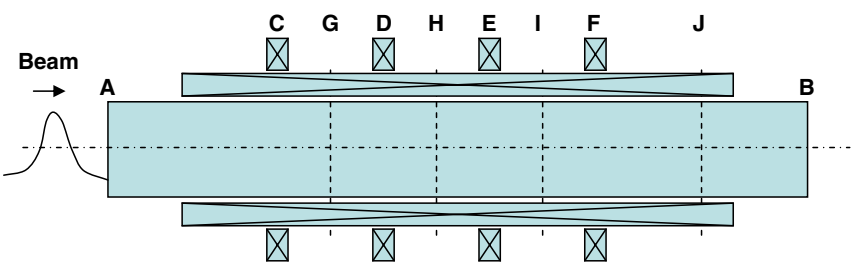

FIG. 8. Geometry of simulation in Sec. IV B. 
$\mathrm{H}, \mathrm{I}$, and $\mathrm{J}$ planes, we also record the fields along the four lines $(x=b, y=0, z=0-10.5 \mathrm{~m} ; x=-b, y=0, z=$ $0-10.5 \mathrm{~m} ; x=0, y=b, z=0-10.5 \mathrm{~m} ; x=0, y=-b$, $z=0-10.5 \mathrm{~m}$ ) to analyze the deflection angle along $z$.

\section{Parameters of injected beam}

The injected beam has a Gaussian temporal profile with standard deviation of $1 \mathrm{~ns}$. Its total charge is $20 \mathrm{nC}$ and the energy is $\mathrm{pc}=5 \mathrm{MeV}$. The transverse distribution is uniform and has a radius of $5 \mathrm{~mm}$. The beam is injected with a horizontal offset of $x=3 \mathrm{~mm}$ and along the $z$ axis.

\section{Simulation results}

The recorded fields along the four lines $(x=b, y=0$, $z=0-10.5 \mathrm{~m} ; x=-b, y=0, z=0-10.5 \mathrm{~m} ; x=0, y=b$, $z=0-10.5 \mathrm{~m} ; x=0, y=-b, z=0-10.5 \mathrm{~m})$ allow a presentation of the virtually measured deflection angle in the horizontal plane along the lattice based on Eqs. (28) and (29), shown in Fig. 9, labeled as "measured $x p$ using axial $B$ dot field." For comparison, the real deflection angle of the beam computed by statistics of the beam is also shown and labeled with " $x p$ of beam centroid." To understand the influence of the nonlocal contribution of the beam current to the axial magnetic field, we also included the plot labeled "smoothed $x p$ of beam centroid," which is based on the convolution of the beam deflection angle with the weighting function $g(z)$. Figure 9 shows a very good agreement between the analytical computation based on Eqs. (28) and (29) and the numerical results. The example demonstrates that the method can be applied also for the case of a fast varying betatron motion, although, at the extreme points (zoomed in Fig. 9), the nonlocal

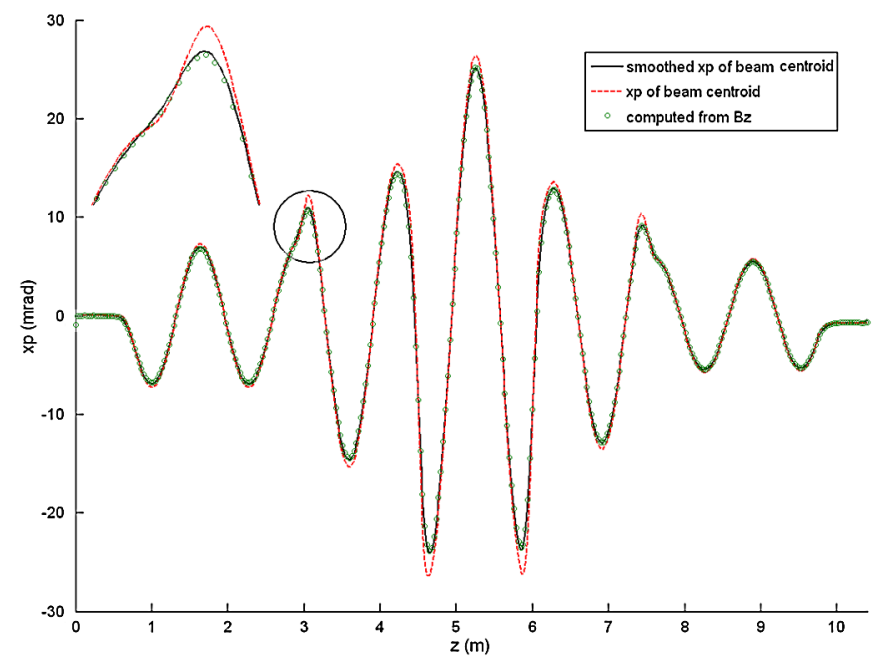

FIG. 9. Comparison between the computed beam deflection angle vs $z$ and real beam deflection angle. The extra curves on the top left are the zoom of the curves in the circle. contribution of the beam current needs to be taken into account to achieve correct results.

\section{DISCUSSION}

The relation between the axial magnetic field and the deflection angle of the beam is derived in two ways. Both methods give very similar results, in one case the nonlocal effects of the beam current are taken into account, in the other case the time dependence is included into the expressions. The analytical methods show that the difference of the axial magnetic fields between the $x+$ and the $x-$ locations is proportional to the deflection angle of the beam in the $y$ - direction, while the $y+$ minus $y$ - field difference returns the beam angle in the $x$ - direction. Both analytical methods enable a direct measurement of the beam deflection angle. A comparison, using numerical simulations based on the MAFIA code, demonstrates a very good agreement between numerical results and the theory.

It should be noted, the axial magnetic field is much smaller than the azimuthal field. In case of a $1 \mathrm{mrad}$ beam deflection angle, the axial magnetic field is only about $0.2 \%$ of the azimuthal magnetic field. One can isolate the axial $B$-dot monitors with azimuthal magnetic field by aligning the loops in one plane vertical to the beam pipe axis with high accuracy. The difference signal of opposite wire-loop antennas will eliminate large parts of the residual contribution of the azimuthal magnetic field. Although the axial magnetic field is weak, it is possible to detect the field. For a typical beam in a linear induction linac, with a peak current of $2 \mathrm{kA}$, a rise time of $20 \mathrm{~ns}$, propagating in a beam pipe of $70 \mathrm{~mm}$ radius, a beam deflection angle of $1 \mathrm{mrad}$ can be detected by a 10 turn wire loop of $1 \mathrm{~cm}^{2}$ area, which generates a $0.6 \mathrm{~V}$ signal, which is not too difficult to be measured precisely.

[1] K. C. Dominic Chan, Carl A. Ekdahl, Yu-Jiuan Chen, and Thomas P. Hughes, in Proceedings of the 20th Particle Accelerator Conference, Portland, OR, 2003 (IEEE, New York, 2003), p. 3210.

[2] W. K. H. Panofsky and M. Bander, Rev. Sci. Instrum. 39, 206 (1968)

[3] K. T. Nguyen, R. F. Schneider, J. R. Smith, and H. S. Uhm, Appl. Phys. Lett. 50, 239 (1987).

[4] K. J. O’Brien et al., Phys. Rev. Lett. 60, 1278 (1988).

[5] R. F. Schneider, K. T. Nguyen, J. R. Smith, and H. S. Uhm, in Proceedings of the 1987 Particle Accelerator Conference (IEEE, Washington, DC, 1987), p. 1054.

[6] H. L. Rutkowski, in Proceedings of LINAC98, Chicago, 1998, p. 21.

[7] D. Villate, Ch. Bonnafond, A. Devin, and E. Merle, in Proceedings of the Particle Accelerator Conference, Dallas, TX, 1995 (IEEE, New York, 1995), p. 2640.

[8] M. Ong, C Avalle, R. Richardson, and J. Zentler, LLNL, Report No. UCRL-JC-125879, 1997. 
[9] Paul Allison and David C Moir, in Proceedings of the Particle Accelerator Conference, Vancouver, BC, Canada, 1997 (IEEE, New York, 1997), p. 1138.

[10] Isaac R. Shokair, Rev. Sci. Instrum. 60, 2969 (1989).

[11] W. W. Rienstra and M. D. Haworth, Rev. Sci. Instrum. 62, 2363 (1991).
[12] R. L. Carlson, R. N. Ridlon, and L.E. Stout, Rev. Sci. Instrum. 57, 2471 (1986).

[13] Alexander Wu Chao, Physics of Collective Beam Instabilities in High Energy Accelerators (Wiley, New York, 1993), p. 6. 